\title{
Intestinal permeabililty in portal hypertension: Still a dilemma
}

\author{
Govind K Makharia
}

Department of Gastroenterology and Human Nutrition

All India Institute of Medical Sciences Ansari Nagar,

New Delhi-110 029, India

Correspondence:

Dr. Govind K Makharia

Email: govindmakharia@gmail.com govindmakharia@aiims.ac.in
Translocation of bacteria and bacterial products from the intestinal lumen into the systemic circulation is a key contributing factor in the pathogenesis of complications of cirrhosis such as spontaneous bacterial peritonitis and hepatorenal syndrome. ${ }^{1-3}$ Three critical factors that facilitate translocation of bacteria in patients with cirrhosis are: alterations in the intestinal microbiota, defective immune system and dysfunction of the intestinal epithelial barrier. .

Translocation of bacterial products, such as endotoxins, across the intestinal epithelium occurs via the transcellular and paracellular routes. Transport of solutes across the transcellular route is carrier-mediated and occurs with the help of transporters and channels. ${ }^{4}$ Transport of molecules through the paracellular route across the intestinal epithelium occurs by the process of diffusion and is not carrier-mediated. An intact intestinal epithelial barrier prevents penetration of the intestinal mucosa by noxious substances such as intestinal microbes and microbial products. Disruption of this barrier system results in a "leaky" intestine, which increases its permeability. The overall function of transport through the intestinal epithelial paracellular route can be assessed functionally by estimating intestinal permeability. ${ }^{5}$

Specialized cell-cell junctional complexes allow selective paracellular transport while maintaining intercellular adhesion (intermediate junctions and desmosomes) and permitting intercellular communication (gap junctions). A tight junction (TJ) at the apico-lateral epithelial surface is potent in restricting the paracellular movement of even very small $(2 \mathrm{kD})$ molecules, thereby preventing the transepithelial transport not only of bacteria, but also of macromolecules such as lipopolysaccharides (LPSs) ${ }^{6-8}$ TJs are made up of several transmembrane and intracytoplasmic proteins. Some of the important transmembranous proteins are: claudins (claudin 2, 3 and 4), occludin and junction adhesion molecule. Some of the intra-cytoplasmic proteins are: ZO-1, ZO-2 and ZO-3, which acts as scaffold between the transmembrane proteins and intracellular actin and myosin filaments. ${ }^{6-8}$ Coordinated interactions of these proteins are important for the regulation and maintenance of $\mathrm{TJ}$ and paracellular permeability. ${ }^{6-8}$

\section{What happens in portal hypertension and cirrhosis?}

Portal hypertension (PHT) can occur because of obstruction to the blood flow through the portal venous system. It is classified as pre-sinusoidal (portal vein block or non-cirrhotic portal fibrosis), sinusoidal (cirrhosis of the liver) and post-sinusoidal (veno-occlusive disease or hepatic venous outflow tract obstruction). ${ }^{9,10}$ PHT in cirrhosis results from increased intrahepatic vascular resistance, caused mainly by the architectural distortion of the liver secondary to formation of fibrous tissue and regenerative nodules, and an increase in portal blood inflow secondary to splanchnic vasodilatation. Splanchnic vasodilatation and the consequent decrease in the effective arterial blood volume lead to activation of neurohumoral systems (i.e. renin-angiotensin-aldosterone system, sympathetic nervous system and antidiuretic hormone), which are responsible for the increased plasma volume. ${ }^{9,10}$ For these 
reasons, portal hypertensive gastropathy or enteropathy generally accompanies PHT. ${ }^{11-13}$ PHT enteropathy may lead to structural changes in the intestinal mucosa in patients with cirrhosis. Macroscopically, the mucosa is oedematous and erythematous because of congestion in the portal venous system. Microscopically, the changes are characterized by the presence of vascular congestion, oedema, fibromuscular proliferation, thickening of the muscularis mucosae, infiltration by chronic inflammatory cells and altered villous/crypt ratio. ${ }^{14}$ ${ }^{16}$ Ultrastructurally, dilatation of the intercellular spaces between neighbouring enterocytes and a reduced number of microvilli have also been described. ${ }^{17}$

Studies have reported a significantly higher gastroduodenal and/or intestinal permeability in patients with cirrhosis who show signs of PHT on endoscopy than in those who do not show such signs. ${ }^{18-20}$ The portal pressure correlates significantly with intestinal permeability, and placement of a transjugular intrahepatic portosystemic shunt has been shown to result in a significant decrease in both the portal pressure and intestinal permeability in patients with cirrhosis. ${ }^{21} \mathrm{In} \mathrm{CCl}_{4}{ }^{-}$ induced cirrhosis models of rats, bacterial translocation (defined as a mesenteric lymph node-positive culture) has been shown to occur in $56 \%$ of those with ascites and $10 \%$ of those without ascites. ${ }^{22}$ Moreover, patients with cirrhosis and bacterial translocation have a higher degree of liver dysfunction (estimated by Child-Pugh score) in comparison with patients without bacterial translocation. ${ }^{23}$ Reiberger et al. ${ }^{24}$ have reported a significant correlation between portal pressure and gastroduodenal and intestinal permeability, plasma levels of LPS-binding protein (LBP) and IL-6 in patients with cirrhosis and PHT. Patients with severe PHT (hepatic venous pressure gradient [HVPG] $>20 \mathrm{mmHg}$ had significantly increased markers of gastroduodenal/intestinal permeability (urine sucrose levels $\mathrm{p}=0.049$; sucrose $/$ mannitol ratios $\mathrm{p}=0.007$; intestinal permeability indices $\mathrm{p}=0.002$ ), and bacterial translocation than those having HVPG $<20 \mathrm{mmHg}$. A significant improvement in intestinal permeability and bacterial translocation was observed after treatment with non-selective beta-blockers in these patients. ${ }^{24}$

A multi-tiered gastrointestinal protective system that includes a protective barrier, intercellular junctions and immune surveillance is critical for the invasion of antigen or microbe through the gastrointestinal tract (GIT). Defects in one or multiple levels of the protective barrier can lead to bacterial translocation through the GIT. Two elements may be relevant in the occurrence of abnormalities in paracellular permeability in patients with cirrhosis-firstly, PHT and secondly the status of liver parenchymal dysfunction, i.e. severity of liver dysfunction. ${ }^{1-3}$ While it is known that severity of liver function abnormalities have a bearing on the intestinal permeability, it is not clear if PHT alone can lead to abnormalities in the paracellular function reflected by an increase in intestinal permeability.

In this issue of Tropical Gastroenterology, Goyal et al. ${ }^{25}$ have tried to answer this question by estimating small intestinal permeability using lactulose/mannitol excretion ratio over a period of 6 hours using 1H-NMR spectroscopy, in 96 patients with PHT (cirrhosis 71, extrahepatic portal vein obstruction [EHPVO] 25). Intestinal permeability was found to be increased in $47(49 \%)$ patients. This increase was more common in patients with cirrhosis than in those with EHPVO (40/71, 56\% vs. 7/25, $28 \%, p=0.01$ ). Patients with abnormal small intestinal permeability had a higher Child-Pugh score, ascites and deranged liver function. On multivariate analysis, presence of cirrhosis, ascites, high serum bilirubin level and high prothrombin time were associated with abnormal small intestinal permeability. The authors concluded that cirrhosis was associated with abnormal small intestinal permeability caused by liver parenchymal dysfunction. They also concluded that PHT per se does not increase intestinal permeability.

While the index study has compared the occurrence of increase in intestinal permeability in patients with cirrhosis and EHPVO and provides some useful information, there are some major concerns about the interpretation of the data.

Around 27\% of patients with EHPVO had increased small intestinal permeability, which should not be regarded as normal. The higher number of patients with cirrhosis having increased small intestinal permeability can be due to their advanced parenchymal dysfunction. What are the reasons for lesser number of patients with EHPVO having abnormal small intestinal permeability? The possible reasons could be the intact immune surveillance of the liver in patients with EHPVO, since the parenchymal liver function and liver architecture remain normal in them. ${ }^{26,27}$ The younger age of patients in the EHPVO group as compared to those with cirrhosis may also be a contributory factor for lower number of patients with EHPVO having abnormal small intestinal permeability. Moreover, bacterial translocation (a clinical correlate of abnormal small intestinal permeability) has been observed in animal models of acute PHT. $^{28}$ 
While there are reasons to expect that PHT alone-without liver parenchymal involvement-can lead to increased small intestinal permeability, the evidence including that provided by Goyal et al. ${ }^{25}$ is not sufficient enough to establish that PHT does not contribute to abnormal small intestinal permeability. The question whether PHT has a role in affecting small intestinal permeability has not been satisfactorily answered. In order to have a definitive answer, more studies are required including patients with non-cirrhotic PHT such as EHPVO and cirrhosis of the liver having normal liver function (cirrhosis with Child A functional status).

Little efforts have gone in addressing the issue of abnormal small intestinal permeability, which is clinically relevant in patients with cirrhosis. The concept developed for the modulation of paracellular permeability in coeliac disease may be extended to abnormal paracelullar permeability in those with cirrhosis. ${ }^{29,30}$ While a gluten-free diet is the standard of care for coeliac disease, the search for an alternative treatment has not stopped. ${ }^{30}$ One strategy has been to prevent the entry of gluten peptides through the TJs by inhibiting a protein called zonulin. Human zonulin, a precursor of prehaptoglobin-2, has homology with a protein zonula occludens toxin (ZOT) expressed by Vibrio cholerae. ${ }^{31}$ Zonulin impairs the epithelial integrity of TJs and regulates epithelial permeability. Zonulin is highly expressed in the intestinal mucosa and blood of patients with coeliac disease. ${ }^{31,32}$ Larazotide acetate (AT-1001, developed by Alba Therapeutics, USA) is an octapeptide derived from a cholera toxin and antagonizes zonulin via receptor blockade. ${ }^{33}$ Therefore, larazotide acetate is expected to reduce the paracellular transport caused by gluten and ameliorate the activation of the pathological immune cascade. The safety of this drug has been established in both phase 1 and 2 clinical trials. A new large (320 patients) phase $2 \mathrm{~b}$ study is ongoing to study different doses $(0.5,1$ and $2 \mathrm{mg}$ per day) of larazotide acetate in patients with coeliac disease (ClinicalTrials.gov identifier: NCT01396213). ${ }^{34}$ Therefore, it will be interesting to explore the expression pattern of zonulin and key TJ proteins in the intestinal mucosa of patients with cirrhosis. Overexpression of zonulin, if present, may open a therapeutic window for prevention of bacterial translocation in patients with cirrhosis.

\section{References}

1. Pijls KE, Jonkers DM, Elamin EE, Masclee AA, Koek GH. Intestinal epithelial barrier function in liver cirrhosis: an extensive review of the literature. Liver Int. 2013 Jul 3. doi: 10.1111/ liv.12271. [Epub ahead of print] PubMed PMID:23879434.

2. Seo YS, Shah VH. The role of gut-liver axis in the pathogenesis of liver cirrhosis and portal hypertension. Clin Mol Hepatol. 2012;18:337-46.

3. Wiest R, Garcia-Tsao G. Bacterial translocation (BT) in cirrhosis. Hepatology. 2005;41:422-33.

4. Bjarnason I, MacPherson A, Hollander D. Intestinal permeability: an overview. Gastroenterology. 1995;108:1566-81.

5. Travis S, Menzies I. Intestinal permeability: functional assessment and significance. Clin Sci (Lond). 1992;82:471-88.

6. Groschwitz KR, Hogan SP. Intestinal barrier function: molecular regulation and disease pathogenesis. J Allergy Clin Immunol. 2009;124:3-20.

7. Berkes J, Viswanathan VK, Savkovic SD, Hecht G. Intestinal epithelial responses to enteric pathogens: effects on the tight junction barrier, ion transport, and inflammation. Gut. 2003;52:439-51.

8. Turner JR. Molecular basis of epithelial barrier regulation: from basic mechanisms to clinical application. Am J Pathol. 2006;169:1901-9.

9. Sanyal AJ, Bosch J, Blei A, Arroyo V. Portal hypertension and its complications. Gastroenterology. 2008;134:1715-28.

10. Sass DA, Chopra KB. Portal hypertension and variceal hemorrhage. Med Clin North Am. 2009;93:837-53.

11. Ohta M, Yamaguchi S, Gotoh N, Tomikawa M. Pathogenesis of portal hypertensive gastropathy: a clinical and experimental review. Surgery. 2002;131:S165-S170.

12. Burak KW, Lee SS, Beck PL. Portal hypertensive gastropathy and gastric antral vascular ectasia (GAVE) syndrome. Gut. 2001;49:866-72.

13. Rondonotti E, Villa F, Signorelli C, de Franchis R. Portal hypertensive enteropathy. Gastrointest Endosc Clin N Am. 2006;16:277-86.

14. Astaldi G, Strosselli E. Peroral biopsy of the intestinal mucosa in hepatic cirrhosis. Am J Dig Dis. 1960;5:603-12.

15. Norman DA, Atkins JM, Seelig LL Jr, Gomez-Sanchez C, Krejs GJ. Water and electrolyte movement and mucosal morphology in the jejunum of patients with portal hypertension. Gastroenterology. 1980;79:707-15.

16. Misra V, Misra SP, Dwivedi M, Gupta SC. Histomorphometric study of portal hypertensive enteropathy. Am J Clin Pathol. 1997;108:652-7.

17. Such J, Guardiola JV, de Juan J, Casellas JA, Pascual S, Aparicio JR, et al. Ultrastructural characteristics of distal duodenum mucosa in patients with cirrhosis. Eur J Gastroenterol Hepatol. 2002;14:371-6.

18. Cariello R, Federico A, Sapone A, Tuccillo C, Scialdone VR, Tiso $\mathrm{A}$, et al. Intestinal permeability in patients with chronic liver diseases: its relationship with the aetiology and the entity of liver damage. Dig Liver Dis. 2010;42:200-4.

19. Di Leo V, Venturi C, Baragiotta A, Martines D, Floreani A. Gastroduodenal and intestinal permeability in primary biliary cirrhosis. Eur J Gastroenterol Hepatol. 2003;15:967-73.

20. Giofré MR, Meduri G, Pallio S, Calandra S, Magnano A, Niceforo $\mathrm{D}$, et al. Gastric permeability to sucrose is increased in portal hypertensive gastropathy. Eur J Gastroenterol Hepatol. 
2000;12:529-33.

21. Xu WH, Wu XJ, Li JS. Influence of portal pressure change on intestinal permeability in patients with portal hypertension. Hepatobiliary Pancreat Dis Int. 2002;1:510-4.

22. Garcia-Tsao G, Lee FY, Barden GE, Cartun R, West AB. Bacterial translocation to mesenteric lymph nodes is increased in cirrhotic rats with ascites. Gastroenterology. 1995;108:1835-41.

23. Cirera I, Bauer TM, Navasa M, Vila J, Grande L, Taurá P, et al. Bacterial translocation of enteric organisms in patients with cirrhosis. J Hepatol. 2001;34:32-7.

24. Reiberger T, Ferlitsch A, Payer BA, Mandorfer M, Heinisch BB, Hayden H, et al, Vienna Hepatic hemodynamic Lab. Nonselective betablocker therapy decreases intestinal permeability and serum levels of LBP and IL-6 in patients with cirrhosis. J Hepatol. 2013;58:911-21.

25. Goyal A, Ghoshal UC, Ahamad I, Roy R, Srivastava D, Mohindra $\mathrm{S}$, et al. Frequency and factors associated with increased small intestinal permeability in patients with portal hypertension. Trop Gastroenterol. 2013;34:136-43.

26. Poddar U, Borkar V. Management of extra hepatic portal venous obstruction (EHPVO): current strategies. Trop Gastroenterol. 2011;32:94-102.

27. Chawla Y, Duseja A, Dhiman RK. Review article: the modern management of portal vein thrombosis. Aliment Pharmacol Ther.
2009;30:881-94.

28. Garcia-Tsao G, Albillos A, Barden GE, West AB. Bacterial translocation in acute and chronic portal hypertension. Hepatology. 1993;17:1081-5.

29. Green PH, Cellier C. Celiac disease. $N$ Engl J Med. 2007;357:1731-43.

30. Sollid LM, Khosla C. Novel therapies for coeliac disease. J Intern Med. 2011;269:604-13.

31. Fasano A, Not T, Wang W, Uzzau S, Berti I, Tommasini A, et al. Zonulin, a newly discovered modulator of intestinal permeability, and its expression in coeliac disease. Lancet. 2000;355:1518-19.

32. Fasano A, Uzzau S, Fiore C, Margaretten K. The enterotoxic effect on zonula occludens toxin on rabbit small intestine involves the paracellular pathway. Gastroenterology. 1997;112:839-46.

33. Paterson BM, Lammers KM, Arrieta MC, Fasano A, Meddings JB. The safety, tolerance, pharmacokinetic and pharmacodynamic effects of single doses of AT-1001 in coeliac disease subjects: a proof of concept study. Aliment Pharmacol Ther. 2007;26:757-66.

34. A double-blind placebo-controlled study to evaluate Larazotide acetate for the treatment of celiac disease. Available at http:// clinicaltrials.gov/show/NCT01396213 (accessed on September $18,2013$. 